\title{
Warfarin related nephropathy: a case report from a tertiary hospital of north India and review of literature
}

\author{
Pannu AK ${ }^{1 *}$, Vohra $\mathbf{G}^{2}$ and Agarwal $\mathbf{P}^{3}$ \\ ${ }^{1}$ Department of Internal Medicine, Post Graduate Institute of Medical Education and Research, Chandigarh, India \\ ${ }^{2}$ Department of Nephrology, Post Graduate Institute of Medical Education and Research, Chandigarh, India \\ ${ }^{3}$ Department of Histopathology, Post Graduate Institute of Medical Education and Research, Chandigarh, India
}

Received: 17 December, 2016; Accepted: 30 December, 2016; Published: 6 January, 2017

*Corresponding author: Ashok kumar Pannu, Department of Internal Medicine, Post Graduate Institute of

Medical Education and Research, Chandigarh, India, Tel no: +919914291115; E-mail: gawaribacchi@gmail.com

\section{Introduction}

Warfarin is the most widely used anticoagulant to treat or prevent thrombotic complications since its approval in the 1950s. Bleeding is the most common complication of warfarin and the risk increases sharply with the International Normalized Ratio (INR). ${ }^{[1]}$

Warfarin Related Nephropathy (WRN) is a newly recognised disease entity and an undiagnosed complication of warfarin. It occurs with over anticoagulation and is associated with increased renal morbidity and all cause mortality. ${ }^{[2]}$

Here we report a case of WRN where warfarin was started on higher initial dose and was continued longer than the recommended duration of anticoagulation. Renal failure improved after cessation of the anticoagulation.

\section{Case description}

This 35 years old lady underwent trans-abdominal hysterectomy and salpingo-opherectomy for uterine fibroid at another hospital. Post operative day seven, patient started having pain and swelling of left lower limb. A compression ultrasound revealed left lower limb Deep Venous Thrombosis (DVT) and she was started on warfarin $7 \mathrm{mg}$ per day along with heparin. Later heparin was stopped and warfarin was titrated according to INR (Table 1).

On $13^{\text {th }}$ November 2016, she came to this hospital with hematuria and uremic symptoms (nausea, vomiting and decreased appetite). Her renal function tests were deranged with serum creatinine of $3.4 \mathrm{mg} / \mathrm{dl}$ (base line creatinine value was not available) and ultrasound showed normal sized kidneys and raised cortical echogenicity. Urine microscopy revealed microscopic hematuria and 1+ albuminuria. Warfarin was stopped, as she had already completed 6 months duration of anticoagulation for provoked DVT.

Further investigations were performed to elucidate the cause of Acute Kidney Injury (AKI)/ Rapidly Progressive Renal Failure (RPRF). Autoimmune markers including antinuclear antibody, anti-neutrophil cytoplasmic antibody and anti-glomerular basement membrane antibody were negative and complements (C3, C4) were normal. Hepatitis B surface antigen, hepatitis C antibody and HIV serology were nonreactive. Hemoglobin A1c (HbA1c) was 6.6\% (>6.5\% is used for diagnosing diabetes).

A kidney biopsy was performed, which included 16 glomeruli with congested capillary tuft. Many of the tubules contained red blood cell cast, white blood cell cast and hyaline cast. Moderate degree of Acute Tubular Necrosis (ATN) was noted and interstitial fibrosis tubular atrophy component occupied about $15 \%$ of core volume. There was mild diffuse interstitial lymphoplasmacytic infiltrate admixed with occasional eosinophils. There was presence of hemosiderin pigment in the interstitium and within tubular epithelial cells indicating hemorrhage. The blood vessels were unremarkable. No deposition of immune complexes or complements was identified on direct immuno-fluorescence. The overall biopsy features were of pigment cast nephropathy, chronic tubule-interstitial nephritis and interstitial hemorrhages consistent with WRN (Figure 1A and 1B). The presence of congested glomerular capillary loops, numerous red blood cell casts in the tubules along with presence of hemosiderin pigment in the interstitium and within tubular epithelial cells signifies glomerular hemorrhage.

Following withdrawal of warfarin, her creatinine started improving (Table 1). On November 24, on a follow up visit,

Table1. INR and serum creatinine trend with warfarin dosing

\begin{tabular}{|c|c|c|c|c|c|c|c|c|c|}
\hline Date & $27 / 4 / 16$ & $23 / 5$ & $25 / 5$ & $22 / 9$ & $25 / 10$ & $29 / 10$ & $31 / 10$ & $3 / 11$ & $24 / 11$ \\
\hline INR & 1.1 & 1.3 & 1.0 & 1.48 & 2.66 & 2.97 & 2.49 & 1.28 & - \\
\hline Warfarin & $7 \mathrm{mg}$ & $7 \mathrm{mg}$ & $7 \mathrm{mg}$ & $7 \mathrm{mg}$ & $5 \mathrm{mg}$ & $3 \mathrm{mg}$ & $3 \mathrm{mg}$ & stopped & - \\
\hline Creatinine & - & - & - & - & - & - & - \\
\hline
\end{tabular}




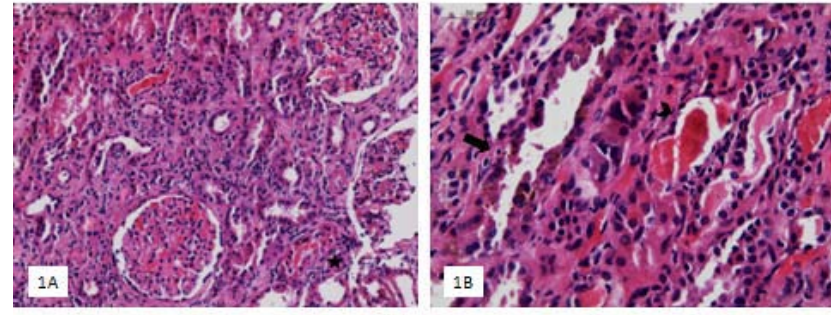

Figure 1: (A)Photomicrograph showing congested glomerular capillary loops and ATN (Black star) (H\&E stain, x200).

1B. Photomicrograph showing numerous RBC casts (Black arrow head) and hemosiderin pigment (Black arrow) within the tubular epithelial cells (H\&E stain, $\mathrm{x} 400)$.

creatinine was improved to $1.3 \mathrm{mg} / \mathrm{dl}$ and no red blood cells (RBCs) were detected on urine microscopy.

\section{Discussion}

WRN is an important differential diagnosis in the evaluation of $\mathrm{AKI} / \mathrm{RPRF}$ in patients on warfarin therapy, especially in the presence of over anti-coagulation and microscopic hematuria. Our patient was started on higher initial dose of warfarin and anticoagulation was continued longer than the recommended duration, which precipitated WRN, however INR value never increased above 3 .

WRN was initially described in patients receiving supratherapeutic doses of warfarin having a distinct pattern of glomerular hemorrhage on renal biopsy. Currently WRN is defined as AKI in the setting of an INR of $>3.0$, excluding other obvious etiologies.

In 2009, Brodsky et al. was first to describe this entity and presented a case series of nine patients of unexplained AKI associated with supra-therapeutic INRs in patients on warfarin. ${ }^{[3]}$ Kidney biopsy was done in every patient and revealed acute tubular injury, glomerular hemorrhage and renal tubular obstruction by RBC cast. Bodsky et al. again undertook a retrospective analysis of the administrative records of 15,258 patients who initiated warfarin therapy between January 2005 and 2008 at the Ohio State University Medical Center. WRN occurred in $20.5 \%$ of the entire cohort, $33.0 \%$ of the Chronic Kidney Disease (CKD) cohort, and $16.5 \%$ of the non-CKD cohort. One-year mortality was $31.1 \%$ in patients with WRN versus 18.9 $\%$ in patients without. ${ }^{[2]}$

The similar kind of nephropathy can occur in any form of over-anticoagulation and the term Anticoagulation Related Nephropathy (ARN) has been proposed after recent reports of AKI with unexplained glomerular hemorrhage associated with over-anticoagulation with dabigatran. ${ }^{[4-6]}$, However, a recent retrospective study of a large Asian cohort found that Dabigatran was associated with a reduced risk of AKI compared with warfarin in atrial fibrillation patients with or without a history of kidney disease. ${ }^{[7]}$

\section{Predisposing Factors and Incidence}

Studied showed that the major risk factor for WRN is CKD, as these patients may be prone to over anticoagulation as spend less time within the target range, required more frequent adjustments and had higher bleeding risk. ${ }^{[8]}$ Other risk factors are advanced age, diabetes mellitus, hypertension, and cardiovascular disease; that is the risk factors of developing AKI. Our patient was incidentally found to have diabetes with HBA1c of $6.6 \%$, which played a part in predisposition of WRN.

The incidence of WRN is difficult to estimate as the available data are largely from retrospective studies published and it appears as a common complication of warfarin. One recent prospective study in elderly patients showed an incidence of $60 \%$. ${ }^{[9]}$

\section{Mechanism of WRN}

The central mechanism for WRN is disruption of the glomerular filtration barrier leads to hemorrhage into Bowman's space and renal tubules followed by formation of RBC casts in the renal tubules, causing obstruction, ischemia, and eventual obliteration. ${ }^{[3]}$ Other proposed mechanism is oxidative stress damage to tubules by RBC without a complete tubular obstruction can contribute WRN. CKD patients have low antioxidant enzyme activities, such as glutathione peroxidase and catalase, which may contribute to a higher risk of WRN. ${ }^{[8]}$ Other possible contributing mechanisms are atheroembolism, interstitial nephritis, apoptosis of glomerular endothelial cells and direct toxic effect of warfarin on kidney. ${ }^{[8]}$

\section{Clinical Consequences and Prevention}

The occurrence of WRN also increased long-term mortality in patients with and without CKD. Brodsky et al. reported that one-year mortality was $31.1 \%$ in the WRN group, $18.9 \%$ in the non-WRN group, with an increased risk of $65 \% \cdot{ }^{[2]}$ An et al. found that the actual mortality rates were $42.8 \%$ with WRN compared with $26.3 \%$ without WRN. The increased risk was highest during 2 years after INR 3.0, reaching $103.8 \%$ at 1 year and $91.9 \%$ at 2 years, and it sharply declined thereafter $\left(50.6 \%\right.$ at 5 years). ${ }^{[10]}$

Treatment of ARN is mainly supportive, and includes cessation of anticoagulation and returning the INR to a therapeutic range. However it is difficult in cases where long term anticoagulation is indicated, as stopping the drug causes risk of thromboembolism, but temporary withdrawal of the drug has resulted in improvement in renal parameters.

Switch over to other anticoagulants may not seem to solve problem as WRN has also occurred in novel oral anticoagulants. ${ }^{[5]}$ However it has been proposed that low dose dabigatran might be associated with a lower risk of ARN than warfarin and may be a safer alternative. ${ }^{[11]}$

$\mathrm{N}$-acetylcysteine, a potent antioxidant, has also been used successfully in animal models to prevent WRN.[12,13] Temporary interruption of anti-coagulation in conjunction with $\mathrm{N}$-acetylcystine has been shown to halt progression of renal dysfunction in WRN. ${ }^{[14]}$ Steroid has been tried for its antiinflammatory action which can occur following glomerular hemorrhage and tubular obstruction in the kidney. ${ }^{[14,15]}$ 


\section{Conclusion}

1. Kidney function including urine output, blood urea, serum creatinine and glomerular filtration rate must be monitored closely in patients receiving oral anticoagulants and the diagnosis of WRN/ARN should be considered in patient with unexplained AKI especially in the presence of over anticoagulation.

2. Renal biopsy should always be considered where WRN is suspected.

3. WRN is a serious complication with a poor renal prognosis and requires the development of successful therapeutic strategies

\section{References}

1. Hylek EM, Singer DE. Risk factors for intracranial hemorrhage in outpatients taking warfarin. Ann Intern Med. 1994;120(1):897-902.

2. Brodsky SV, Nadasdy T, Rovin BH, Satoskar AA, Nadasdy GM, Wu $\mathrm{HM}$, et al. Warfarin-related nephropathy occurs in patients with and without chronic kidney disease and is associated with an increased mortality rate. Kidney Int. 2011;80(2):181-189. doi: 10.1038/ ki.2011.44

3. Brodsky SV, Satoskar A, Chen J, Nadasdy G, Eagen JW, Hamirani M et al. Acute kidney injury during warfarin therapy associated with obstructive tubular red blood cell casts: a report of 9 cases. Am J Kidney Dis. 2009;54(6): 1121-1126. doi: 10.1053/j.ajkd.2009.04.024

4. Kadiyala D, Brewster UC, Moeckel GW. Dabigatran induced acute kidney injury. Paper presented at: The American Society of Nephrology Annual Meeting; November 1-4, 2012; FR-P01122, San Diego, California

5. Ryan M1, Ware K, Qamri Z, Satoskar A, Wu H, Nadasdy G, et al. Warfarinrelated nephropathy is the tip of the iceberg: direct thrombin inhibitor dabigatran induces glomerular hemorrhage with acute kidney injury in rats. Nephrol Dial Transplant. 2014;29(12):2228-2234. doi: $10.1093 / \mathrm{ndt} / \mathrm{gft} 380$

6. Brodsky SV, Hebert LA. Anticoagulant-related nephropathy: Is an AKI elephant hiding in plain view? J Am Coll Cardiol. 2016;68(21):22842286. doi: 10.1016/j.jacc.2016.09.926.
7. An JN, Ahn SY, Yoon CH, Youn TJ, Han MK, Kim S, et. al. The occurrence of warfarin- related nephropathy and effects on renal and patient outcomes in korean patients. PLoS One. 2013;8(4):e57661. doi:10.1371/journal.pone.0057661.

8. Yang Y, Liu T, Zhao J, Li G. Warfarin-related nephropathy: prevalence, risk factors and prognosis. Int J Cardiol. 2014; 176(3):1297-1298. doi: 10.1016/j.ijcard.2014.07.166.

9. Lim AK, Campbell DA. Haematuria and acute kidney injury in elderly patients admitted to hospital with supratherapeutic warfarin anticoagulation. Int Urol Nephrol. 2013;45(2):561-570. doi: 10.1007/ s11255-012-0364-0.

10. An JN, Ahn SY, Yoon CH, Youn TJ, Han MK, Kim S, et al. The occurrence of warfarin-related nephropathy and effects on renal and patient outcomes in Korean patients. PLoS ONE. 2013;8(4):e57661. doi: 10.1371/journal.pone.0057661.

11. Chan YH, Yeh YH, See LC, Wang CL, Chang SH, Lee HF, et. al. Acute kidney injury in Asians with atrial fibrillation treated with dabigatran or warfarin. J Am Coll Cardiol. 2016;68(21):2272-2283. doi: 10.1016/j. jacc.2016.08.063.

12. Ozcan A, Ware K, Calomeni E, Nadasdy T, Forbes R, Satoskar AA, et al. 5/6 nephrectomy as a validated rat model mimicking human warfarin-related nephropathy. Am J Nephrol. 2012;35(4):356-64. doi: $10.1159 / 000337918$.

13. Ware K1, Qamri Z, Ozcan A, Satoskar AA, Nadasdy G, Rovin BH, et al. $\mathrm{N}$-acetylcysteine ameliorates acute kidney injury but not glomerular hemorrhage in an animal model of warfarin-related nephropathy. Am J Physiol Renal Physiol 2013; 304(12):F1421-7. doi: 10.1152/ ajprenal.00689.2012.

14. Ng CY, Tan CS, Chin CT, Lim SL, Zhu L, Woo KT, et al. Warfarin related nephropathy: a case report and review of the literature. BMC Nephrol. 2016;17:15. doi: 10.1186/s12882-016-0228-4.

15. Iqbal HI; Akbar S. Warfarin related nephropathy in the absence of supratherapeutic INR. AJKD. 2014;63(5):B58. doi:http://dx.doi. org/10.1053/j.ajkd.2014.01.176. 